\title{
Crafting a Research Article from Dissertation: the Arduous Final Hurdle
}

\author{
Debajyoti Mohanty
}

Received: 13 January 2015 / Accepted: 16 January 2015 / Published online: 1 February 2015

(C) Association of Surgeons of India 2015

I have read the article comparing the outcome of minor anorectal surgeries under local anesthesia (LA) versus spinal anesthesia (SA) by Kulkarni et al. [1] with interest. The anorectal ailments indeed account for bulk of the patients reporting for surgical consultation. Favoring LA in the surgical management of these patients can tremendously cut down the requirement for general and spinal anesthesia in our already overburdened operation theaters.

In this modern era of evidence-based medicine, randomized control trials (RCT) are considered the benchmark for comparing the outcome of different treatment modalities. The inherent strength of RCT design lies in the unbiased and unpredictable allocation of patients into the different intervention arms with the aim to abolish baseline imbalances. In this article, the authors have disclosed neither the basis of selection of a sample size of 60 patients nor the technique of randomization employed for enrollment of patients into the two intervention arms. The fairness of their randomization process is also under suspicion as the duration of symptoms was $\leq 6$ months in all the $30(100 \%)$ patients of the LA group but in only $12(40 \%)$ patients of the SA group. The article is crammed with 12 colorful diagrams and 14 tables making it quite difficult to focus on the relevant outcome measures. In fact, there is duplication of presented data as both the figures and the tables depict the same set of observations. Reference to all the tables and figures is cited in the introduction while they should have been ideally incorporated in the results. There are many glaring

D. Mohanty $(\bowtie)$

Department of General Surgery, All India Institute of Medical

Sciences, Raipur, GE Road, Tatibandh Raipur,

Chhattisgarh 492099, India

e-mail: debajyoti.mohanty@gmail.com inconsistencies in the presented data that merit clarification by the authors. The mean age of the patients was mentioned as $45.97 \pm 16.46$ years in the text but $41.35 \pm 12.25$ years in table 1 . Similarly, the least presenting symptom was mass per rectum in the text, but in table 3, constipation was identified as the least presenting symptom. The mean duration of symptoms was mentioned as $11.4 \%$, whereas it should have been mentioned in days or months. The authors have variably reported the incidence of constipation in their patients ( 1 in table 3 and 36 in table 5). Only patients with grade II and III hemorrhoids are included as per the mentioned study protocol but the results showed $2.9 \%$ patients with grade IV hemorrhoids. The follow-up period was also different in the table (6 months) and in the text (12 months).

Prima facie, it appears that this article has been hastily crafted from a postgraduate dissertation. The publication of the research work carried out during postgraduation period is highly rewarding for the resident and should be encouraged. Ideally, the research article should be a neatly packaged miniature form of the dissertation. Precise analytical representation of the relevant outcome measures in sync with the aim of the study while leaving out the insignificant observations is the key for converting the thesis into a good research article.

Source of Funding Nil.

Conflict of Interest None.

\section{Reference}

1. Kulkarni SV, Agarwal P, Nagraj AK (2014) To compare the outcome of minor anorectal surgeries under local anesthesia versus spinal anesthesia. Indian J Surg 76(5):343-349 